\title{
Assessment of vehicles headway during queue dissipation at signal-controlled intersection under mixed traffic
}

\author{
Satyajit Mondal and Ankit Gupta* \\ Department of Civil Engineering, Indian Institute of Technology (Banaras Hindu University), Varanasi 221005 , India
}

The present study focuses on the comprehensive analysis of vehicles headway at signalized intersections under mixed traffic stream. Data were collected at five signalized intersections from three different cities (Allahabad, Delhi and Chandigarh) of India. The discharge headway of vehicles was analysed after the onset of green signal when the initial queue dissipated through the intersection. A box-and-whisker plot was generated for discharge headway of vehicles to quantify its equitable profile. The plot delineated that headway of vehicles decreased with the initial queue dispersion. Although the differences between headway of vehicles were almost immutable after the fifth queue position, gives a saturation headway of $2.05 \mathrm{sec} /$ vehicle. The headway distribution at each queue position was also evaluated using various continuous distribution models. The Kolmogorov-Smirnov (K-S) test was performed to define the adequate distribution model. The statistical results obtained from the $\mathrm{K}-\mathrm{S}$ test indicated that the discharge headway distribution at each queue position followed a log-normal distribution. Multilinear regression technique was applied to analyse and develop a model for discharge headway using green signal time and vehicle type as independent variables. The result indicated that longer green time had a significant impact on discharge headway. The outcomes of this study may be beneficial in the microsimulation model development for signalized intersections to assess their performance.

Keywords: Discharge headway, mixed traffic, regression analysis, signalized intersection, queue dissipation.

INTERSECTIONS are a crucial part of any road network and significantly affect the efficiency of the entire transportation system ${ }^{1}$. Discharge characteristics during the onset of green signal are fundamental parameters to evaluate the performance of a signalized intersection ${ }^{2}$. Headway of vehicles is a crucial measure, put emphasis on discharge flow estimation at signalized intersection ${ }^{3,4}$. Vehicles headway is the time difference of two successive vehicles with respect to some reference point (stop line for signalized intersections $)^{5}$. The headway of a vehicle standing

*For correspondence. (e-mail: ankit.civ@iitbhu.ac.in) in front of the queue is measured as the time between the vehicle crossing the stop line and the initiation of green signal. Consequently, headways of the vehicles standing in the queue are measured as the time difference between the crossing of stoppage line (considered as reference line) of preceding vehicle and subject vehicle. The rear wheels of a vehicle are considered as a reference line over the stop line to measure its headway. According to the Highway Capacity Manual (HCM 2010) ${ }^{5}$, a vehicle standing in front of the queue would take more time to react while the signal changes from red to green. This reaction time is minimized with every subsequent vehicle standing in the queue and a vehicle's headway decreases gradually until it becomes a constant (Figure 1). This stabilized headway begins from the fourth or fifth vehicle position when the vehicles have fully accelerated to cross the stop line. This phenomenon is known as discharge headway of vehicles. HCM 2010 suggests that saturation flow can be estimated directly using the constant discharge headway value known as saturation headway ${ }^{5}$.

Figure 1 depicts a lane-wise homogeneous traffic condition with proper traffic regulations. In the case of heterogeneous traffic stream, a combination of both slow and fast moving vehicles travel through the same stretch without any segregation. Also, the static (vehicle dimension) and dynamic (speed, acceleration, power) characteristics of motorized vehicles have a significant impact on the discharge headway, where motorized two-wheelers and three-wheelers usually stand in front of the queue and can move in the gaps available between larger vehicles. This makes the discharge less disciplined and thus it is challenging to measure the headway value. Several studies have focused on homogeneous traffic conditions, whereas limited efforts have been made to analyse discharge headway under heterogeneous traffic conditions. Therefore, the present study analyses vehicle discharge headway for least lane discipline mixed traffic stream at signalized intersections.

\section{Literature review}

An accurate discharge headway may provide an adequate estimation of discharge flow and reasonable design of a 


\section{RESEARCH ARTICLES}

signalized intersection. A significant effort has been made in the last few decades to analyse the discharge headway of vehicles during queue dissipation through the intersection in green time. Initially, the discharge headway for the first five vehicles in the queue was analysed and the average headway value reported ${ }^{6}$. Researchers from various countries have studied the discharge headway characteristics for different traffic and geometric conditions. They found that discharge headway displays significant changeability for different locations due to change in both traffic and geometric conditions ${ }^{7,8}$. Bonneson $^{9}$ developed a model to analyse the discharge headway using several parameters such as driver reaction time, vehicle speed and acceleration. He found that the stabilized headway can only be obtained after the eighth and ninth vehicle positions in a queue. Several researchers have analysed the headway distribution for different vehicle positions in a queue under different traffic flow conditions $^{10-12}$. Jin et al. ${ }^{10}$ found that discharge headway for vehicles standing in subsequent queue positions followed a log-normal distribution, although a vehicle standing in front of the queue followed a normal distribution. They proposed a car-following model to describe the specific distribution of a vehicle's headway. Studies have also reported that in free-flow condition the headway follows a log-normal distribution, whereas in congested conditions the vehicle's headway is more suited to loglogistic distribution during peak hours ${ }^{11,12}$. Researchers have analysed queue discharge characteristics such as vehicle headway, speed and acceleration at signalized intersections under mixed traffic conditions. Vehicle's headway showed a decreasing trend with increase in queue position as the initial lost time is getting minimized with the queue position ${ }^{13}$. As a result, the clearing speed of queued vehicles increased with increase in the position of vehicles in the queue. Although the headway of a vehicle showed a decreasing trend with increase in the queue position, after the 12 th vehicle position, the discharge headway showed an increasing trend. Also, the headway of vehicle through lane stream exhibited an elongation tendency with optimal discharge speed

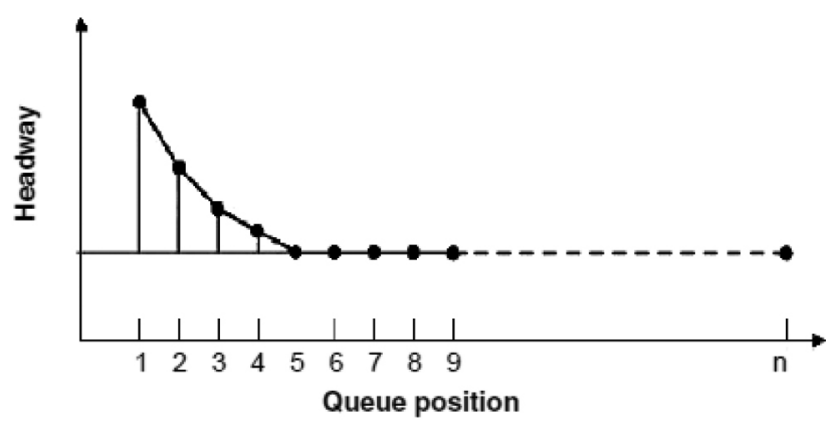

Figure 1. Discharge headway during queue dissipation according to Highway Capacity Manual (HCM) 2010. of $27 \mathrm{kmph}$ for the lowest average headway ${ }^{14}$. Digital countdown display had a significant impact on discharge characteristics of vehicles. A number of studies have analysed the effect of digital signal countdown timers on the performance of the signalized intersections ${ }^{15-17}$. They found that the mean headway for all vehicle positions had a smaller value for the countdown intersection, which gave a higher significant value of saturation flow rate.

It can be seen from the literature that many researchers have pointed out that headway of vehicles shows a decreasing trend with increase in the queue position and the headway value is stabilized after the fourth or fifth vehicle position. HCM 2010 provided a steady headway of $1.9 \mathrm{sec}$ after its stabilization ${ }^{5}$. However, each study in the literature gave a different headway value, as it varies in several roadway, traffic and control conditions ${ }^{16}$. Also, most of the studies were based on homogeneous and lanedisciplined traffic condition where the traffic stream was mainly dominated by cars. A limited number of studies have reported the headway value and its distribution at each queue position under heterogeneous traffic conditions. The present study analyses discharge headway of departing vehicles through a signalized intersection under mixed traffic conditions.

\section{Traffic data collection and extraction}

Field data collection for the present study was more challenging for the heterogeneous traffic stream with less lane disciplined flow. During the red signal, smaller-sized vehicles like two-wheelers and three-wheelers are able to move through the gap between large-sized vehicles and stand in the front of the queue, making a number of haphazard streamlines. During the green signal, vehicles move through the intersection together and randomly depending upon the gap available between them. This makes it more difficult to identify the vehicles that are following each other. In order to get an accurate headway value, it is required to collect data from a vantage point so that the movement of every vehicle can be traced correctly. The recording was done to trace the vehicular movement through the intersection by installing a video camera at a higher elevation near the selected site.

Table 1. Values of various parameters of selected sites

\begin{tabular}{llccc}
\hline Site no. & Location & $\begin{array}{c}\text { Width of } \\
\text { approach }(\mathrm{m})\end{array}$ & $\begin{array}{c}\text { Cycle } \\
\text { length (s) }\end{array}$ & $\begin{array}{c}\text { Cycle } \\
\text { analysed }\end{array}$ \\
\hline I-1 & Allahabad & 7.13 & 122 & 77 \\
I-2 & & 8.95 & 145 & 75 \\
I-3 & Delhi & 10.2 & 170 & 51 \\
I-4 & & 10.2 & 170 & 40 \\
I-5 & Chandigarh & 7.70 & 120 & 44 \\
\hline
\end{tabular}




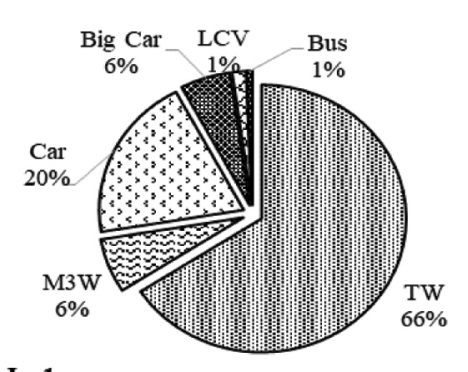

I - 1

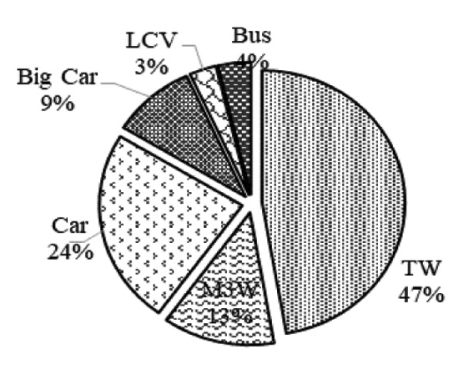

I - 2

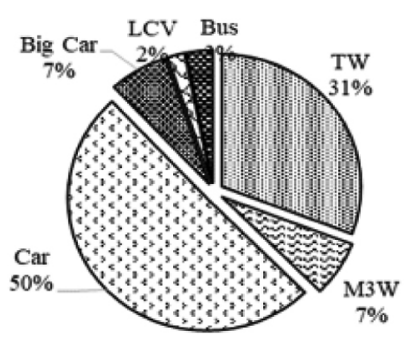

I - 3
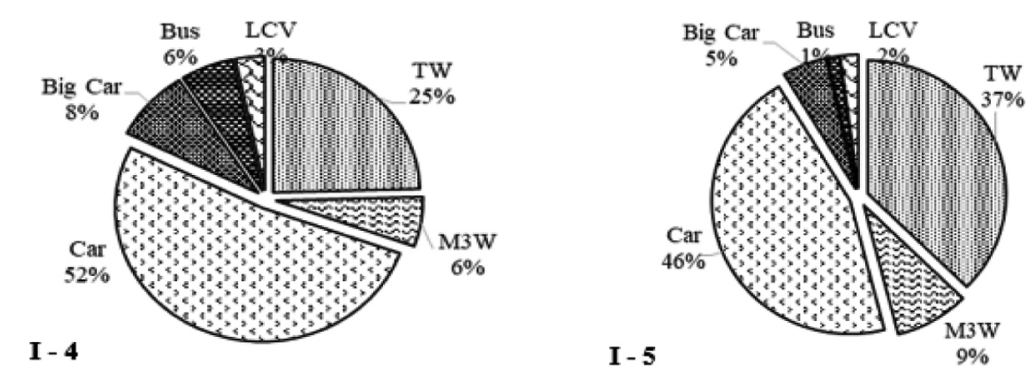

Figure 2. Vehicular composition at all the selected intersections.
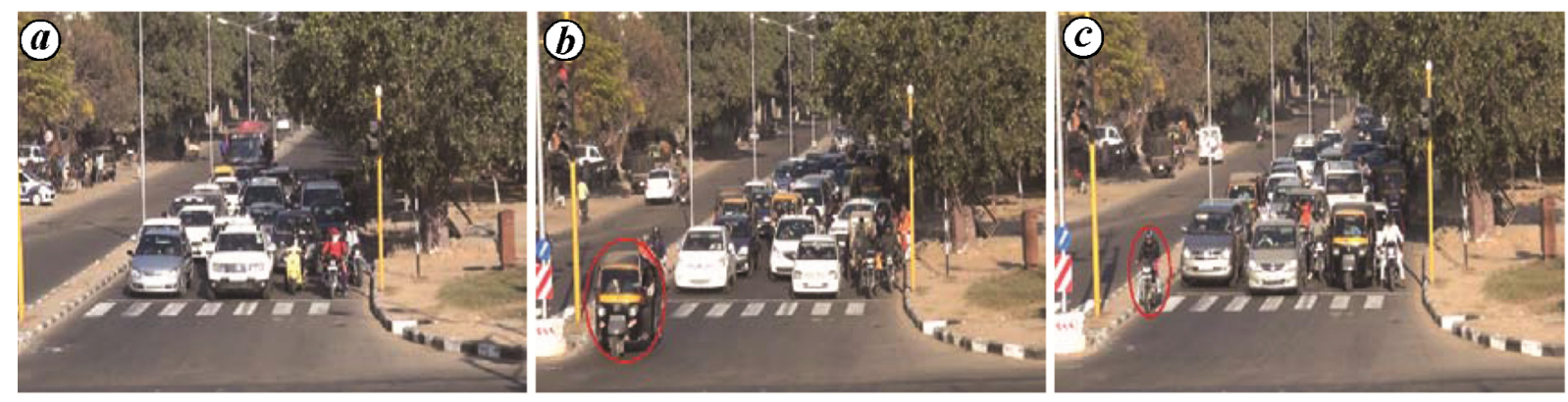

Figure 3. Vehicle stoppage position $(\boldsymbol{a})$ before and $(\boldsymbol{b}, \boldsymbol{c})$ after the approach stop line during red interval.

Initially, three different cities were chosen for traffic data collection. All the selected sites have a channelized section for left-turn movement with less pedestrian influence at the intersection. The traffic data collection was carried out in the morning and evening peak hours. Table 1 provides the values of various parameters for each selected site.

The existing traffic was categorized into six vehicle classes, i.e. car, big car (BC), motorized two-wheeler (TW), motorized-three-wheeler (M3W), bus and light commercial vehicle $(\mathrm{LCV})^{18}$. The compositional share of each vehicle class was extracted from the collected video data (Figure 2). Also, the discharge headway of vehicles was extracted with respect to the stop line (marking on the pavement surface) at the intersection. In all the selected intersections, vehicles usually stood before the stop line during the red signal. Although, in some cases, vehicles (generally TW and M3W) stood after the stop line during the red signal. Such vehicles were not considered in the headway data analysis. Figure 3 depicts such conditions with vehicles standing before and after the stop line.

\section{Discharge headway characteristics}

Discharge headway is a vital traffic measure widely used in the evaluation of various performance measures in traffic studies $^{19,20}$. An appropriate estimation of discharge flow can be done using the accurate value of discharge headway leading to a proper evaluation of capacity at a signalized intersection. Figure 4 illustrates a fundamental discharge headway profile of vehicles after the onset of green signal $^{21}$.

The discharge headway showed a decreasing trend until it reached a stabilized interval. At the onset of green signal, the discharge headway of some vehicles was higher as they needed extra time to perceive the signal variations. The headway of consecutive vehicles progressively decreased until it reached a constant (saturation headway) till the initial queue dissipated. The discharge headway evaluation for mixed traffic stream was more complicated due to the haphazard movement of vehicles during the onset of green signal. Vehicles generally move in an irregular manner according to their physical 
dimension and make a number of streamlines without following the preceding vehicle. Consequently, estimation of saturation headway is more difficult for the existing traffic stream. The saturation headway can be estimated using eq. (1) as

$$
h_{\mathrm{s}}=\frac{\sum_{i=n}^{m} h_{i}}{(m-n+1)}
$$

where $h_{\mathrm{s}}$ is the saturation headway (sec/vehicle), $n$ denotes the vehicle position in the queue at the start of the saturation region and $m$ represents the position of the last vehicle of the initial queue.

All the video data were processed to extract vehicular headway value during the onset of green signal. Discharge headway for individual vehicles in the queue was analysed by considering the approach stop line as a reference point. Table 2 enlists the headway statistics up to the tenth vehicle position. A box-and-whisker plot was generated using the mean discharge headway value (Figure 5).

Figure 5 shows that headway of vehicles decreases with the initial queue dispersion. It also indicates that the differences between headway values of vehicles are almost immutable after the fifth vehicle position of the initial queue. Thus, saturation headway of $2.05 \mathrm{sec} /$ vehicle was estimated using eq. (1).

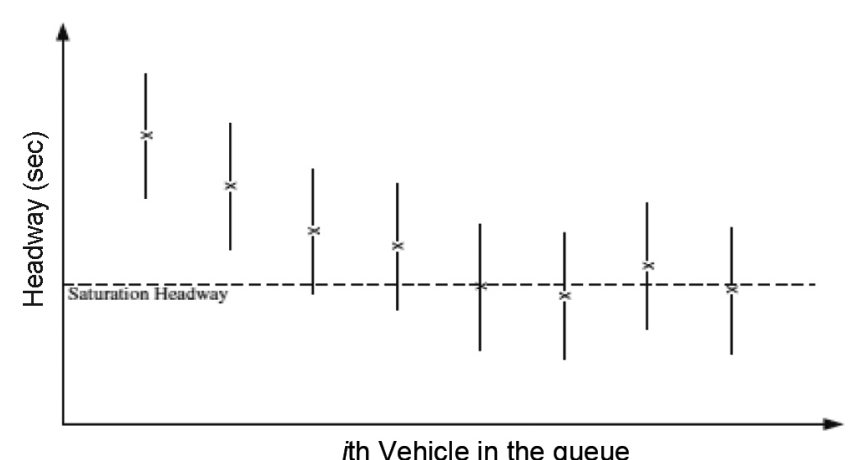

Figure 4. Conceptual discharge headway pattern of vehicles ${ }^{21}$.

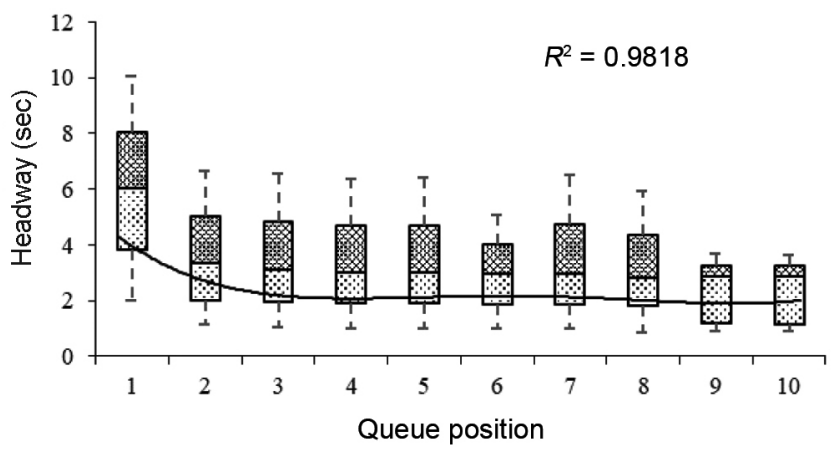

Figure 5. Discharge headway pattern of vehicles.
The saturation flow can be estimated using eq. (2) as

$$
S=\frac{3600}{h_{s}}
$$

where $S=1756$ vehicle/h.

\section{Discharge headway distribution}

The driving behaviour of a mixed traffic stream is tedious to evaluate due to the presence of a variety of vehicles with different driving styles ${ }^{22,23}$. Vehicles headway and its distribution is an important parameter to understand the driving behaviour in order to estimate several traffic stream parameters and formulate a suitable policy for mixed traffic stream ${ }^{24}$. The initial queue formed during the red signal was considered for headway analysis, whereas vehicles join in green were omitted. It was perceived that an initial queue contained maximum 15 entirely stopped vehicles. Therefore, headway analysis was carried out up to the tenth queue position for each initial queue formed during the red signal. Table 3 shows the descriptive statistics of discharge headway for each queue position.

The table illustrates that a positive skewness is observed with different mean and median values at each queue position (from Q1 to Q10). This phenomenon is noticed due to the presence of a variety of vehicles in the traffic stream and the different driving styles. As a result, the suitability of normal distribution digresses for headway distribution for each queue position due to the random arrival of a vehicle type. Consequently, several types of continuous distributions such as normal distribution, exponential distribution, logistic distribution, log-logistic distribution, Log-normal distribution, Erlang distribution and Weibull distribution were tested. The appropriate headway distribution was selected according to the statistical significance of observed headway value for each queue position. A statistical Kolmogorov-Smirnov (K-S) test was performed to define the adequate distribution model by quantifying the $\mathrm{K}-\mathrm{S}$ statistics between empirical distribution of headway data and cumulative distribution of reference distribution. Also, the statistical hypothesis test for each distribution was carried out to check the suitability using the $\mathrm{K}-\mathrm{S}$ test. The statistical results obtained using the $\mathrm{K}-\mathrm{S}$ statistical analysis show that the discharge headway distribution at each queue position follows a log-normal distribution (Figure 6).

The basic function of a log-normal distribution is represented as

$$
f(x)=\frac{1}{x \sigma \sqrt{2 \pi}} \exp -\frac{1}{2}\left(\frac{\ln (x)-\mu}{\sigma}\right)^{2}, x>0,
$$

where $\sigma$ and $\mu$ are the scale and location parameters respectively.

CURRENT SCIENCE, VOL. 116, NO. 3, 10 FEBRUARY 2019 
RESEARCH ARTICLES

Table 2. Headway value at each queue position

\begin{tabular}{lcccccccccc}
\hline Position & Q1 & Q2 & Q3 & Q4 & Q5 & Q6 & Q7 & Q8 & Q9 & Q10 \\
\hline Mean & 4.01 & 2.55 & 2.25 & 2.16 & 2.16 & 2.04 & 2.10 & 2.06 & 1.94 & 1.95 \\
SD & 0.74 & 0.68 & 0.68 & 0.69 & 0.61 & 0.63 & 0.76 & 0.77 & 0.75 & 0.68 \\
Sample size & 320 & 320 & 320 & 320 & 320 & 312 & 308 & 300 & 292 & 276 \\
\hline
\end{tabular}

Table 3. Statistical results of the discharge headway

\begin{tabular}{lccccccc}
\hline Position & $\begin{array}{c}\text { Sample } \\
\text { size }\end{array}$ & $\begin{array}{c}\text { Mean } \\
(\mathrm{sec})\end{array}$ & $\begin{array}{c}\text { Minimum } \\
(\mathrm{sec})\end{array}$ & $\begin{array}{c}\text { Maximum } \\
(\mathrm{sec})\end{array}$ & $\begin{array}{c}\text { Median } \\
(\mathrm{sec})\end{array}$ & $\begin{array}{c}\text { Variance } \\
(\mathrm{sec})\end{array}$ & $\begin{array}{c}\text { Skewness } \\
(\mathrm{yes} / \mathrm{no})\end{array}$ \\
\hline Q1 & 320 & 4.01 & 1.93 & 7.73 & 3.95 & 0.99 & $1.03(\mathrm{Y})$ \\
Q2 & 320 & 2.67 & 1.08 & 5.03 & 2.9 & 0.61 & $0.57(\mathrm{Y})$ \\
Q3 & 320 & 2.45 & 0.92 & 4.8 & 2.1 & 0.54 & $0.52(\mathrm{Y})$ \\
Q4 & 320 & 2.31 & 1.04 & 5.01 & 2.08 & 0.53 & $0.71(\mathrm{Y})$ \\
Q5 & 320 & 2.23 & 1.01 & 4.22 & 2.05 & 0.42 & $0.54(\mathrm{Y})$ \\
Q6 & 312 & 2.25 & 1.04 & 4.96 & 2.02 & 0.56 & $0.93(\mathrm{Y})$ \\
Q7 & 308 & 2.23 & 1.01 & 4.82 & 2.01 & 0.56 & $0.56(\mathrm{Y})$ \\
Q8 & 300 & 2.17 & 1.00 & 4.92 & 2.01 & 0.61 & $0.72(\mathrm{Y})$ \\
Q9 & 292 & 2.16 & 1.01 & 4.11 & 2.02 & 0.49 & $0.45(\mathrm{Y})$ \\
Q10 & 276 & 2.06 & 1.01 & 4.48 & 1.99 & 0.43 & $0.62(\mathrm{Y})$ \\
\hline
\end{tabular}

$\mathrm{Y}$ indicates the positive skewness in headway values at each queue position.
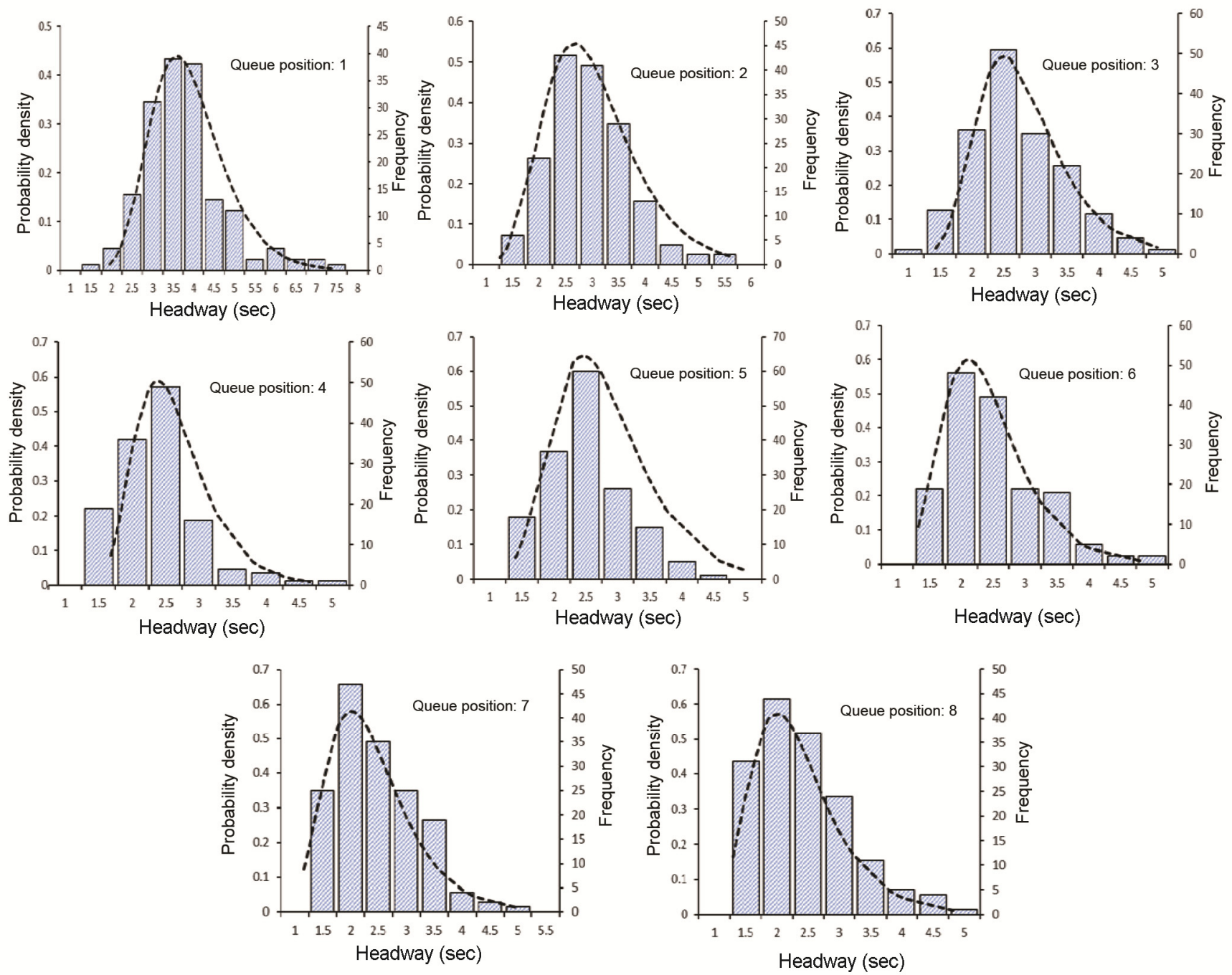

Figure 6. Probability density function of vehicles headway at each queue position. 
Table 4. Results of statistical test of discharge headway

\begin{tabular}{ccccc}
\hline & & \multicolumn{3}{c}{ Kolmogorov-Smirnov } \\
\cline { 3 - 5 } Position & Sample size & Statistics & $P$ value & Critical value $(\alpha=0.05)$ \\
\hline 1 & 320 & 0.038 & 0.59 & 0.044 \\
2 & 320 & 0.011 & 0.39 & 0.044 \\
3 & 320 & 0.013 & 0.59 & 0.044 \\
4 & 320 & 0.014 & 0.18 & 0.044 \\
5 & 320 & 0.015 & 0.19 & 0.044 \\
6 & 312 & 0.013 & 0.43 & 0.044 \\
7 & 308 & 0.014 & 0.45 & 0.044 \\
8 & 300 & 0.014 & 0.18 & 0.045 \\
9 & 292 & 0.016 & 0.21 & 0.046 \\
10 & 276 & 0.014 & 0.17 & 0.046 \\
\hline
\end{tabular}

Table 5. Multilinear regression modelling for both the regimes

\begin{tabular}{|c|c|c|c|c|c|c|}
\hline \multirow[b]{2}{*}{ Factor } & \multicolumn{3}{|c|}{ Regime $1\left(R^{2}=0.43, \mathrm{SEE}=0.84\right)$} & \multicolumn{3}{|c|}{ Regime $2\left(R^{2}=0.49, \mathrm{SEE}=1.02\right)$} \\
\hline & Coefficient & $P$ value & $t$-statistic & Coefficient & $P$ value & $t$-Statistic \\
\hline Intercept & 3.69 & 4.0E-188 & 33.25 & 1.81 & $7.78 \mathrm{E}-21$ & 9.54 \\
\hline Green time & -0.05 & $5.0 \mathrm{E}-70$ & -18.52 & 0.04 & $5.40 \mathrm{E}-20$ & 9.33 \\
\hline TW & -0.73 & $1.1 \mathrm{E}-11$ & -6.84 & -0.51 & 4.9E-04 & -3.49 \\
\hline M3W & -0.57 & $2.4 \mathrm{E}-06$ & -4.73 & -0.51 & $3.48 \mathrm{E}-03$ & -2.93 \\
\hline Car & -0.51 & $1.4 \mathrm{E}-06$ & -4.84 & -0.55 & $3.62 \mathrm{E}-06$ & -4.65 \\
\hline Big car & -0.39 & $1.1 \mathrm{E}-03$ & -3.28 & -0.65 & $1.49 \mathrm{E}-03$ & -3.18 \\
\hline
\end{tabular}

Table 4 shows the statistical results obtained for the log-normal distribution for each queue position. Thus, the null hypothesis can be accepted according to the statistical significance.

\section{Variation of discharge headway: a multilinear regression approach}

A number of influencing factors affecting the discharge headway have been highlighted by various researchers $^{16,17,20}$. Models were developed based on their prevailing roadway, traffic and control conditions. The present study analyses the variation of discharge headway with signal timing (green) and the type of vehicle travelling through an intersection (right turn and through movements) during the green signal. Figure 7 is a box plot generated for the discharge headway. It shows two distinctive regimes: (a) the headway values show a decreasing trend until they become stabilized and (b) the headway of vehicle is increasing as the initial queue departs and less vehicles are present to use remaining green time. Also, drivers travelling through the intersection at the end of green signal do not have much idea about the remaining time of green due to the non-countdown signal system. Most of the previous studies have analysed the discharge headway until it reached a stable level (up to 10-12 vehicles in a queue). However, the second regime shows that the discharge headway may increase at the end of the green signal due to the absence of vehicles. This implies that longer green signal time has a significant impact and may be considered in the modelling of discharge headway.

The linear regression model was fitted with both the regimes; Figure 7 shows the results. Values given in brackets are the $P$-values and $t$-statistics of the coefficient at $95 \%$ level of confidence. In the first regime, the headway value decreased at a rate of 0.0496 times per unit change in green signal time. Whereas the next regime showed an increasing trend at the rate of 0.0328 times. The effect of vehicle type was also analysed within the regimes using multi-linear regression analysis, where discharge headway was considered as the dependent variable, while green signal time and vehicle type were considered as independent variables. Equation (4) shows the generalized form of regression analysis between the dependent and independent variables.

$$
\begin{aligned}
D_{s}= & \text { Intercept }+\alpha_{\mathrm{GT}} \times \mathrm{GT}+\alpha_{\mathrm{TW}} \times \mathrm{TW}+\alpha_{M 3 W} \\
& \times M 3 W+\alpha_{\mathrm{car}} \times \mathrm{car}+\alpha_{\mathrm{BC}} \times \mathrm{BC},
\end{aligned}
$$

where $D_{\mathrm{s}}$ is the departure headway, GT the green signal time (sec), TW, M3W and $\mathrm{BC}$ represent two-wheeler, motorized three-wheeler and big car respectively.

The values of the intercept and coefficient of the independent variables were determined at a higher coefficient of regression and lower standard error of estimation. Bus 
and LCV statistically insignificant to the discharge headway; therefore they were considered as independent variables in the model. Table 5 shows the results of multilinear regression analysis for both the regimes.

The intercept value in regime 1 indicates the headway of a vehicle standing in the front of the queue at the initiation of green signal. The coefficient values of the independent variables in regime 1 show a negative impact on discharge headway as the headway value decreases and becomes stabilized at the end of regime 1 . In regime 2 , the green signal time has a positive impact on discharge headway as longer green signal time may increase the headway value. Although the negative coefficients for vehicle type imply that the appearance of any vehicle in that duration reduces the headway value. Figure 8 shows the proportional values of the coefficient.

The figure shows the proportional values of the coefficients for individual variables for both regimes. Among all the variables, green signal time shows an extensive change over both the regimes starting from a negative impact in regime -1 , indicating that the headway value decreases to a positive effect in regime 2 (indicating that the headway value increase towards the end of the green signal time). Also, the coefficients of vehicles show a significant result. In regime 1, TW has the highest coefficient, as in the Indian mixed traffic stream TWs generally stand at the front of the queue and maintain a

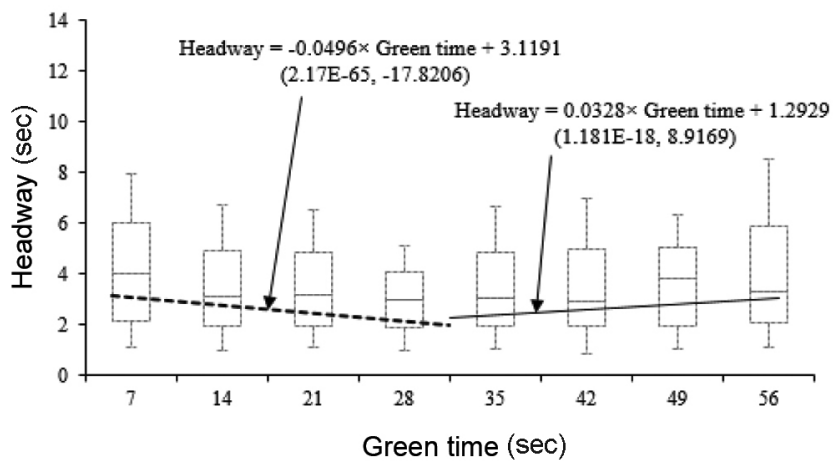

Figure 7. Variation of discharge headway with green time.

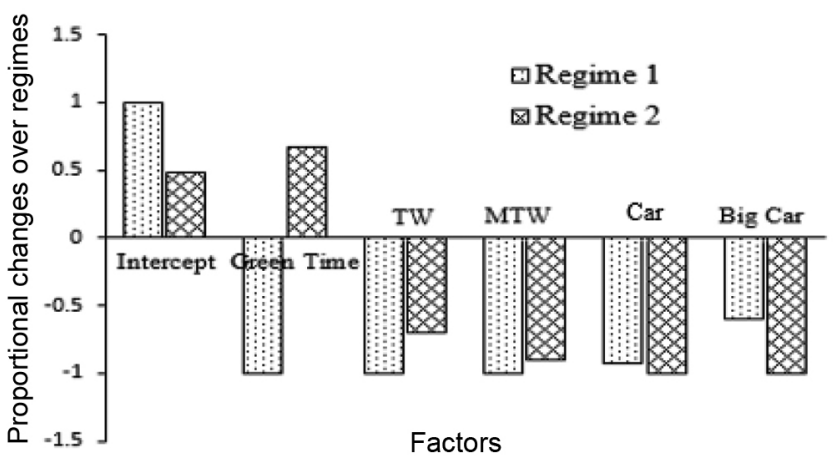

Figure 8. Proportional changes in coefficient values. lower gap during discharge time. Whereas, the $\mathrm{BC}$ has the lowest coefficient as large-sized vehicle maintain the longer gap at the initiation of green signal. In regime 2, TW has the lowest coefficient while BC has the highest coefficient. At the end of the green signal, vehicles travel with higher speed. Therefore, the four wheeled vehicle has the lower headway value than the motorized two wheeler or three wheeler due to higher acceleration characteristics.

Few studies have analysed discharge headway characteristics at signalized intersections, especially for mixed traffic stream. Most of them have recommended certain distribution functions, i.e. Gumbel, log-normal, Weibull, gamma and exponential for discharge headway analysis based on the traffic situations ${ }^{10,25-27}$. The present study also adopts various continuous distribution functions and finds that $\log$-normal distribution is the most suited to represent discharge headway distribution at each queue position (up to the tenth position). We have also developed a model to find the discharge headway value using green signal time and vehicle type as the two parameters having a significant effect on headway value. Radhakrishnan and Ramadurai ${ }^{28}$ formulated discharge headway model incorporating various influencing parameters such as vehicle type, lateral position (median, middle and near kerb) and green signal time using linear mixed effect regression for Indian mixed traffic stream ${ }^{28}$. In a traffic stream where vehicles do not follow the lane discipline, it is difficult to identify their positions due to haphazard movements. Therefore, the model developed in this study may be efficient to analyse headway characteristics at signalized intersections for non-lane-based mixed traffic stream.

\section{Conclusion}

The present study focuses on a comprehensive analysis of discharge headway at signalized intersections under mixed traffic conditions. Data were collected at five different signalized intersections from three different cities of India. The discharge headway of vehicles was is analysed after the onset of green signal, and the initial queue dissipated through the intersection. A box-andwhisker plot was generated for discharge headway of vehicles to quantify its equitable profile. The plot shows that headway of vehicle decreases with the initial queue dispersion. Although the differences between headway vehicles are almost immutable after the fifth queue position, gives a saturation headway of $2.05 \mathrm{~s} /$ vehicles. The headway distribution at each queue position was also evaluated using various continuous distribution models. The $\mathrm{K}-\mathrm{S}$ test was performed to define the adequate distribution model. The statistical results obtained from the $\mathrm{K}-\mathrm{S}$ statistical analysis showed that the distribution of discharge headway at each queue position followed a 


\section{RESEARCH ARTICLES}

log-normal distribution. The multi regime is fitted with the headway distribution during the entire green signal period. Vehicle type and green signal time were the significant factors affecting discharge headway. Multilinear regression analysis was used to develop a model of discharge headway by considering vehicle type and green signal time as independent variables. The result showed that in regime 1 the headway value decreased with the increase in green signal time and presence of a vehicle type. In regime 2 , the headway value increased with the increase in green signal time, and reduced due to the appearance of a vehicle type. The outcome of this study may be beneficial in the microsimulation model development for signalized intersections to assess their performance.

However, for a better understanding of discharge headway and its distribution, more data need to be collected and factors such as roadway geometry factors, driver behaviour, acceleration, deceleration and speed characteristics of vehicles have to be considered to improve the model performance.

1. Biswas, S., Chakraborty, S., Ghosh, I. and Chandra, S., Saturation flow model for signalized intersection under mixed traffic condition. Transp. Res. Rec.: J. Transp. Res. Board, 2018; https://doi. org $/ 10.1177 / 0361198118777407$.

2. Mondal, S. and Gupta, A., Discharge characteristics analysis of queued-up vehicles at signal controlled intersections. Int. J. Civ. Eng., 2018; https://doi.org/10.1007/s40999-018-0343-7.

3. Al-Ghamdi, A., Analysis of time headways on urban roads: case study from Riyadh. J. Transp. Eng., ASCE, 2001, 127(4), 289294.

4. Brackstone, M., Waterson, B. and McDonald, M., Determinants of following headway in congested traffic. Transp. Res. Part F, 2009, 2, 131-142

5. Highway Capacity Manual, Transportation Research Board of the National Academics, Washington, DC, USA, 2010, 5th edn.

6. Greenshields, B. D., Schapiro, D. and Ericksen, E. L., Traffic performance at urban street intersections. Bureau of Highway Traffic Technical Report No. 1, Yale University, New Haven, Conn, USA, 1947

7. Moussavi, M. and Tarawneh, M., Variability of departure headways at signalized intersections. In Compendium of Technical Papers, Annual Meeting - Institute of Transportation Engineers, Orlando, FL, USA, 1990, pp. 313-317.

8. Al-Ghamdi, A., Entering headway for through movements at urban signalized intersections. Transp. Res. Rec.: J. Transp. Res. Board, 1999, 1678, 42-47.

9. Bonneson, J. A., Study of headway and lost time at single-point urban interchanges. Transp. Res. Rec.: J. Transp. Res. Board, 1992, 1365, 30-39.

10. Jin, X., Zhang, Y., Wang, F., Li, L., Yao, D., Su, Y. and Wei, Z., Departure headways at signalized intersections: a log-normal distribution model approach. Transp. Res. Part C, 2009, 17(3), 318-327.

11. Yin, S., Li, Z., Zhang, Y., Yao, D., Su, Y. and Li, L., Headway distribution modeling with regard to traffic status. In IEEE Intelligent Vehicles Symposium, Xi'an, China, 2009, pp. 1057-1062.
12. Wu, J., Hu, J. and Sun, J., Departure headway distribution modeling for ordinary large cities based on video information. In 5th International Conference on Computer Sciences and Convergence Information Technology, Seoul, South Korea, 2010, pp. 1040 1045.

13. Dey, P. P., Nandal, S. and Kalyan, R., Queue discharge characteristics at signalized intersections under mixed traffic conditions. Eur. Transp., Transp. Euro., 2013. 55(8), 1-21.

14. Gao, L. and Alam, B., Optimal discharge speed and queue discharge headway at signalized intersections. In 93rd Annual Meeting of Transportation Research Board, CD-ROM, Washington, DC, United States, 2014.

15. Ibrahim, M. R., Karim, M. R. and Kidwai; F. A., The effect of digital count-down display on signalized junction performance. Am. J. Appl. Sci., 2008, 5(5), 479-482.

16. Sharma, A., Vanajakshi, L. and Rao. N., Effect of phase countdown timers on queue discharge characteristics under heterogeneous traffic conditions. Transp. Res. Rec.: J. Transp. Res. Board, 2012, 2130, 93-100.

17. Devalla, J., Biswas, S. and Ghosh, I., The effect of countdown timer on approach speed at signalized intersections. Proc. Comput. Sci., 2015, 52, 920-925.

18. Indian Highway Capacity Manual (Indo-HCM), CSIR-Central Road Research Institute, New Delhi, 2017.

19. Arasan, T. V. and Koshy, R. Z., Headway distribution of heterogeneous traffic on urban arterials. J. Inst. Eng., 2003, 84, 210 215 .

20. Zhang, G., Wang, Y., Wei, H. and Chen, Y., Examining headway distribution models with urban freeway loop event data. Transp. Res. Rec.: J. Transp. Res. Board, 2007, 1999, 141-149.

21. Limanond, T., Chookerd, S. and Roubtonglang, N., Effects of countdown timers on queue discharge characteristics of through movement at a signalized intersection. Transp. Res. Part C, 2009, 17(6), 662-671.

22. Hagring, O., Estimation of parameters in distribution of headways in roundabouts. J. Transp. Eng., ASCE, 2002, 128, 403-411.

23. Dey, P. and Chandra, S., Desired time gap and time headway in steady-state car-following on two-lane roads. J. Transp. Eng., ASCE, 2009, 135, 687-693.

24. Moridpour, S. and Aliakbari, M., Headway distributions in urban highways under heavy traffic conditions. J. Geotech. Transp. Eng., 2016, 2(2), 39-45.

25. Hung, W. T., Tian, F. and Tong, H. Y., Discharge headway at signalized intersections in Hong Kong. J. Adv. Transp., 2002, 37(1), 105-117.

26. Murat, Y. S. and Gedizlioglu, E., Investigation of vehicle time headways in Turkey. Proc. Inst. Civ. Eng.-Transp., 2007, 160(2), 73-78.

27. Yun, M. and Huang, L., Distribution characteristics of headway at weaving section of signalized intersection upstream. In Proceedings of the Fifth International Conference Transport Engineering, ASCE, Dalian, China, 2015, pp. 1346-1353.

28. Radhakrishnan, S. and Ramadurai, G., Discharge headway model for heterogeneous traffic conditions. Transp. Res. Proc., 2015, 10, $145-154$.

Received 24 September 2018; revised accepted 12 November 2018

doi: $10.18520 / \mathrm{cs} / \mathrm{v} 116 / \mathrm{i} 3 / 437-444$ 